\title{
Protoplanetary disks and the dawn of planets with SKA
}

\author{
L. Testi ${ }^{* 1,2,3}$, L. Perez ${ }^{4}$, I. Jimenez-Serra ${ }^{2}$, M. Hoare ${ }^{5}$, A. Boley ${ }^{6}$, T.L. Bourke ${ }^{7}$, J.R. \\ Brucato $^{1}$, P. Caselli ${ }^{8}$, C. Chandler ${ }^{4}$, C. Codella ${ }^{1}$, A. Isella ${ }^{9}$, J. Lazio ${ }^{10}$, M.E. \\ Palumbo $^{11}$, L. Podio ${ }^{1}$, A. Remijan ${ }^{4}$, J. Tarter ${ }^{12}$, D.J. Wilner ${ }^{13}$ \\ ${ }^{1}$ INAF-Osservatorio Astrofisico di Arcetri, Firenze, Italy; ${ }^{2}$ European Southern Observatory, \\ Garching, Germany; ${ }^{3}$ Excellence Cluster Universe, Garching, Germany; ${ }^{4}$ National Radio \\ Astronomy Observatory, USA, ${ }^{5}$ University of Leeds, Leeds, UK; ${ }^{6}$ University of British \\ Columbia, Canada; ${ }^{7}$ SKA Organization, Jodrell Bank, UK; ${ }^{8}$ Max Planck Institut fuer \\ Extraterrestrische Physik, Garching, Germany; ${ }^{9}$ Rice University, Houston TX, USA; ${ }^{10}$ Jet \\ Propulsion Laboratory, California Institute of Technology, Pasadena, CA; ${ }^{11}$ INAF-Osservatorio \\ Astrofisico di Catania, Catania, Italy; ${ }^{12}$ SETI Institute, Mountain View CA, USA; \\ ${ }^{13}$ Harvard-Smithsonian Center for Astrophysics, Cambridge MA, USA \\ E-mail: ltearcetri.inaf.it, ltesti@eso.org
}

\begin{abstract}
Protoplanetary disks are formed as a consequence of angular momentum conservation during star formation. Planetary systems are expected to form within the disks during the first few Myrs of evolution of the system. The SKA1, in combination with ALMA and E-ELT, can provide unique constraints on the physical and chemical processes associated with planet formation. In this contribution we investigate the key areas in which SKA1 and its future extensions will advance our understanding of planetary system formation and the delivery of complex and pre-biotic molecules on the proto-planets. We focus mainly on three aspects: the growth of solids and the first steps towards the rocky cores of planets, the detectability of pre-biotic molecules in the gas phase in disks, and the effects of the central star on the disk chemistry. For each area, we discuss which SKA1 science requirements need to be fullfilled to allow this science to be carried out. It emerges that, if the high frequency bands are deployed, SKA1 has the potential of delivering unique and transformational science in the field of planet formation. Key science contributions are also possible with a SKA1 without Band 5, but the impact will be significantly reduced. We also identify the limitations of SKA1 and the expansion of capabilities that will be required in the future.
\end{abstract}

Advancing Astrophysics with the Square Kilometre Array

June 8-13, 2014

Giardini Naxos, Italy

\footnotetext{
* Speaker.
} 


\section{Introduction}

Disks form as a consequence of angular momentum conservation during the star formation process (e.g. Shu et al. 1987). In the early stages of the star formation process the disk mediates accretion from the parental core to the forming protostar, while at later stages it provides the natural location and material for the planet formation process. The observational study of the properties and evolution of protoplanetary disks is thus a direct probe of the initial conditions for planet formation. The presence of protoplanetary disks around young stars was originally inferred from the emission in excess to the stellar photosphere at infrared and submillimetre wavelengths (eg. Beckwith et al. 1990; Beckwith \& Sargent 1991). It was very rapidly understood that the excess emission was likely due to a disk-like distribution of dust located around and heated by the young star at the centre. This was also consistent with the dynamics of the molecular gas, which resembled Keplerian rotation around the central object. The inferred sizes (up to few times $100 \mathrm{AU}$ in radius, corresponding to a few arcsec at the distance of the nearby star forming regions) and masses (up to $10-20 \%$ of the central star mass) were also consistent with the expected values for a pre-solar nebula. These ideas were then spectacularly confirmed by the HST silhouette optical absorption images a few years later (O'dell et al. 1993). Following these initial observations, protoplanetary disks around young solar analogs have been extensively studied in nearby star forming regions. The recent review by Williams \& Cieza (2011) summarizes most of our current knowledge of the general properties of protoplanetary disk populations in nearby star forming regions.

Due to the connection with planet formation, the physical and chemical evolution of protoplanetary disks are of fundamental importance to understand the formation of planetary systems in general and of our own Solar System in particular. Most of the research in the last few years has focused in understanding the global disk population properties and evolution with mass and environment and the evolution of dust and gas under the competing effects of planet formation and photoevaporation from the central star.

The study of protoplanetary disks is one of the main science drivers of ALMA, JWST and the E-ELT. These facilities will advance significantly our understanding of the physics of disks and planet formation, especially in combination with longer wavelength radio observations. The SKA1 will enable unique observations for constraining the physics of planet formation and disk dissipation mechanisms. Studies of disks in the frequency range below $15 \mathrm{GHz}$ have been severely hampered by the limited sensitivity of existing radio facilities. The upgraded VLA is providing tantalizing results that show the potential of SKA1. Wilner (2004) discussed in some detail the case for observing protoplanetary disks with the SKA, here we provide an extension of that case, taking into account the advances in the last decade and especially considering the most recent observational results at millimetre and submillimetre wavelengths. We discuss detailed sensitivity limits, frequency ranges, and spectral resolution requirements for SKA1 and its future extensions, as well as observational strategies and constraints for important new experiments in this field.

In the continuum, the main emission mechanisms are related to three separate phenomena that can be disentangled by monitoring observations at different wavelengths in the $1-15 \mathrm{GHz}$ range: thermal emission from dust, thermal emission from ionized winds, and strongly variable thermal and non-thermal emission arising close to the stellar surface from accretion and magnetic activity. Key SKA observations of protoplanetary disks will focus on (1) the growth of dust grains towards 
planetesimals, which can be constrained only by gaining access to centimetre-wave emission from large dust grains and pebbles, (2) the disk cold gas component and its possible pre-biotic molecular species, and (3) the disk-star interaction traced by the emission of photoevaporative and disk winds, which regulate angular momentum transport in disks and the origin of outflows, and of energetic stellar flares that may drive some of the chemical processing of both solid and gaseous material in the disk. Based on detailed models of the physics of protoplanetary disks, we discuss the required sensitivity, angular resolution and time cadence of programmes aimed at addressing these questions.

For spectral lines, emission from complex and pre-biotic molecules are potentially detectable in protoplanetary disks at the high end of SKA1 frequency range. Given the cold conditions of disk midplanes, organics can be locked into the icy mantles of dust grains and remain undetected. However, cosmic ray induced secondary uv-photons can desorb ice in heavily shielded regions, as recent Herschel measurements of water in cores and protoplanetary disks suggest (Hogerheijde et al. 2011; Caselli et al. 2012; Podio et al. 2013). This process would allow for the release of organic molecules in the gas phase. We discuss model computations of the expected emission within the SKA1 bands from the amino acid Glycine desorbed from icy mantles in disks. We conclude that detection may be possible, albeit not easy with SKA1, paving the way for abundance studies of this important pre-biotic molecule.

\section{Grain growth in disks}

Grain settling and growth in protoplanetary disks is thought to be the initial step of the formation of the rocky cores of planets (see e.g. Beckwith et al. 2000). In the last decades, the processes of collision and sticking of grains have been studied theoretically and in laboratory experiments (eg. Blum \& Wurm 2008), and are used in global disk evolution models to describe the physics of dust evolution (eg. Birnstiel et al. 2012). The recent review of Testi et al. (2014) provides a summary of the status of our theoretical, experimental and observational understanding of the grain growth process in disks. In Figure 1 we show a sketch of a protoplanetary disk with the physical processes related to grain growth and an indication of the regions that can be probed with current and future observing facilities.

The main observational signature for the growth of grains in the disks midplane is the spectral index of the dust opacity coefficient, which can be reliably measured once the thermal structure of the disk is properly modeled and contamination from other sources of continuum emission (e.g. from electrons in winds) is properly taken into account (Testi et al. 2003, 2001). The combination of sensitive millimetre and centimetre observations of the nearby TW Hya disk disentangle emission mechanisms and provide strong evidence for pebble-sized grains (Pascucci et al. 2012; Wilner et al. 2005). Results from recent millimetre wave surveys of the continuum emission from disks have shown that grain growth is a widespread phenomenon, also occurring very early in the lifetime of disks (eg. at the disk formation stage; Miotello et al. 2014) and also, somewhat unexpectedly, in exotic systems (eg. brown dwarf disks; Ricci et al. 2012, 2014). These results, if confirmed, would suggest that the early growth of planetary cores, and perhaps full planets, is a fast process in protoplanetary disks. 

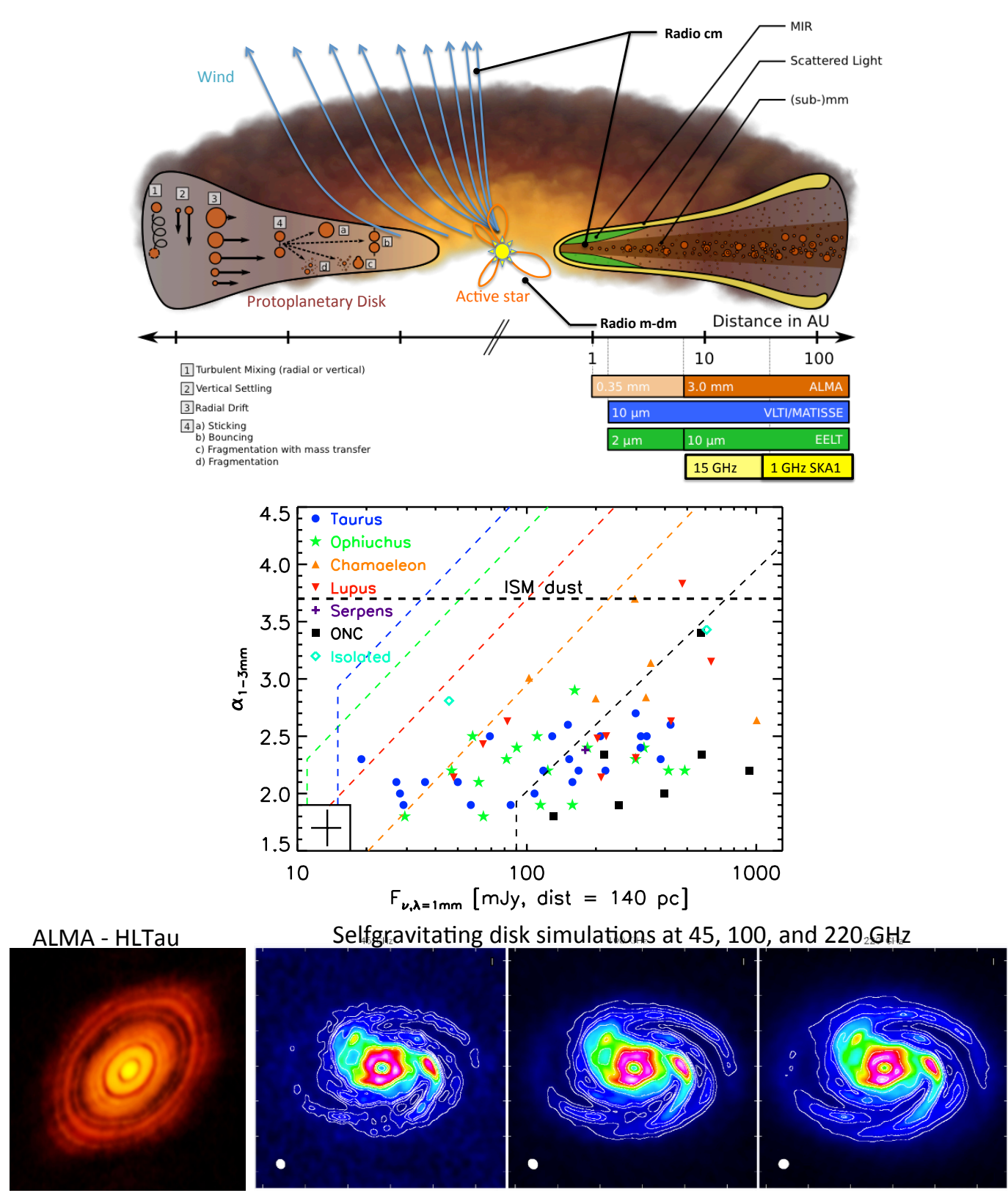

Figure 1: Observing grain growth in disks, from models to observations. Top panel: sketch of a diskstar system, indicated are the main sources of continuum emission in the thermal infrared, submillimetre and radio domains; the physical processes for grain growth in the disk and the scales probed by various facilities for disks in nearby star forming regions are also shown. Middle panel: surveys of the 1 to $3 \mathrm{~mm}$ spectral index versus $1 \mathrm{~mm}$ flux of disks in nearby star forming regions; small values of the spectral index correspond to grain growth, the value expected for the interstellar medium (ISM) grains is shown; all observations have been scaled to a common distance of 140 pc. Bottom: the leftmost panel is the ALMA-SV dust emission image at $230 \mathrm{GHz}$ of the HL Tau disk, at a spatial resolution of approximately $5 \mathrm{AU}$, the ring features in the outer disk likely represent dust aimuthal concentrations; the other panels show simulated disk images for an angular resolution of $\sim 0.15$ " at 45, 100 and $220 \mathrm{GHz}$ (from left to right), the theoretical disk has been located at a distance of $50 \mathrm{pc}$ from the Sun, corresponding to the closest known protoplanetary disk system (TW Hya). The two top panels have been adapted and modified from Testi et al. (2014), the bottom panels have been adapted from Dipierro et al. (2014) and from http://www. eso.org/public/news/eso1436/. 
Models have difficulties in explaining these findings, unless some form of grain trapping or localized growth is occurring in the disk (Pinilla et al. 2012). The future observational work will thus be focused on trying to spatially resolve grain growth in disks. Observations at $\mathrm{cm}$-wavelengths play a key role as they allow us to trace solids grown to pebble sizes across the $\mathrm{cm}$-size barriers, this process is expected to be very localized in protoplanetary disks. A key and unique contribution of SKA1 will be the ability to detect and resolve spatially the wind emission from the disk/star, which needs to be carefully accounted for and removed to study the dust properties. Initial results have been and are being obtained combining data from millimetre interferometers (including ALMA) with data from the JVLA at $0.7,1.3$ and $6 \mathrm{~cm}$. Pérez et al. (2012) found convincing evidence for a radial stratification of the dust properties in protoplanetary disks, confirming earlier suggestions (Banzatti et al. 2011; Guilloteau et al. 2011). The angular resolution and surface brightness sensitivity to detect localized growth is just becoming available for the outer disk regions with ALMA. A dramatic demonstration of the power of high angular resolution onservations to study the dust distribution in disks has just been obtained by the ALMA Science Verification observations of HL Tau, shown in Fig. 1, bottom panel. Note that ALMA will not be able to provide the angular resolution to resolve these structures at its longest operating wavelengths, which will instead be made possible with SKA1.

In Figure 1 we show an example of a simulated disk with spiral arms in the gas distribution, in this case caused by gravitational instability. The higher gas densities in the arms provide local pressure maxima that could very effectively trap large dust particles. The simulated ALMA observations show that high angular resolution (of the order or better than $0.1^{\prime \prime}$ is critical to detect the expected features in these disks (Cossins et al. 2010; Dipierro et al. 2014).

\subsection{Dust evolution with SKA1}

The main contribution of SKA1 to the study of dust evolution will be to extend with a significant improvement in sensitivity and resolution the work done by JVLA in combination with ALMA. During the early science phase, the first key step will be to produce sensitive multifrequency surveys of disks in nearby star forming regions at a few arcsecond resolution. Those young star clusters that are inaccessible to the JVLA in particular can be surveyed for the first time in the 1-14 GHz range. Combined with ALMA surveys that are and will be carried out in the coming years, these will allow to separate the dust emission from the contaminating wind emission in the SED and to provide robust statistics of the timescale for grain growth in protoplanetary disks, which is still lacking. This will be an essential milestone to constrain the timescales for the formation of the rocky cores of planets.

With the completed SKA1-MID it will be possible to map the grain growth as a function of radius with a resolution of few $\mathrm{AU}$ on selected bright disks in nearby star forming regions. These requirements are well captured in the current version of the SKA1 science requirements (Braun (2014), in particular for the continuum sensitivity near 1 and $12 \mathrm{GHz}$ ).

\subsection{Dust evolution beyond SKA1}

With the full SKA it will be possible to routinely map grain growth at even higher resolution and on wide range of targets. To fully resolve planet formation and probe the physics of grain 
trapping and growth, it is necessary to obtain an angular resolution comparable to the disk scale height, which is roughly $10 \%$ of the distance from the central star. This implies that within $10 \mathrm{AU}$, where the relevant planet formation region is occurring, the angular resolution has to be of the order or better than $1 \mathrm{AU}$. At the distance of the nearby star forming regions this corresponds to 10 mas resolution and the required surface brightness sensitivity will be tens of $\mathrm{K}$ in the continuum. These figures will define the key requirements for this science beyond SKA1.

\section{Complex organic molecules in disks}

Complex organic molecules are produced in the interstellar medium, most efficiently through solid state chemistry channels. The "ices" in dense interiors of molecular clouds represent a location where atoms and molecules are packed close together and partly shielded from the dissociative interstellar radiation field. For the simple organic $\mathrm{H}_{2} \mathrm{CO}$, there are already indications from resolved morphology and low excitation in disks that the predominant formation pathway is hydrogenation of CO ice followed by desorption (Qi et al. 2013). How far the chemistry of complex organic molecules and pre-biotic molecules goes in the interstellar medium is an important area to be understood in order to figure out the complexity of the material that is delivered on the planetary surfaces. There is convincing evidence for the presence of simple amino acids in meteorites and possibly in comets of our own Solar System (Pizzarello et al. 1991; Glavin et al. 2006; Elsila et al. 2009), laboratory experiments have also shown that complex molecules and simple amino acids like Glycine $\left(\mathrm{NH}_{2} \mathrm{CH}_{2} \mathrm{COOH}\right)$ can form in irradiated interstellar ice analogs (Muñoz Caro et al. 2002; Holtom et al. 2005; Modica \& Palumbo 2010).

The recent detection of water vapour in the prestellar core L1544 shows that even in the cold interior of these dense cores, cosmic rays yelding secondary high energy radiation can desorb a measurable quantity of molecules from the ices (Caselli et al. 2012). Jiménez-Serra et al. (2014) have shown that not only water, but also complex pre-biotic molecules (like Glycine) may be detectable if desorbed together with the water molecules (see Figure 2 for the predictions of Glycine emission in the prestellar core L1544). We expect that this result can be extended to the cold midplanes of protoplanetary disks (see Figure 2 bottom panel). The direct detection in the gas phase and measurements of the abundance with respect to water of complex organic molecules would be a significant milestone in studying our cosmic heritage and the ability of ISM and disk chemistry to produce the raw material required for the development of life on exoplanets. As complex organic molecules are easily destroyed in the warm molecular layers of disks, which are exposed to the heating radiation from the star, the best hope to detect and study them is in the cold interior of disks, where they may be desorbed from ices as outlined. In these cool conditions, the rotational spectra of complex molecules is significantly skewed towards low frequencies. As a consequence the detection with ALMA is less easy and limited to the (as of today unfunded) Band 1 and 2 covering the 35-80 GHz range. The high frequency SKA band with its superb sensitivity can play a major role in detecting and studying complex organic molecules.

\subsection{Detection of complex organic molecules in disks with SKA1}

The requirements to observe complex organic molecules in protoplanetary disks imply that it will be necessary to reach at least $\sim 50 \mathrm{mK}$ sensitivity for narrow lines $(\Delta \mathrm{V} \sim 0.2 \mathrm{~km} / \mathrm{s})$ at $\sim 3^{\prime \prime}$ 

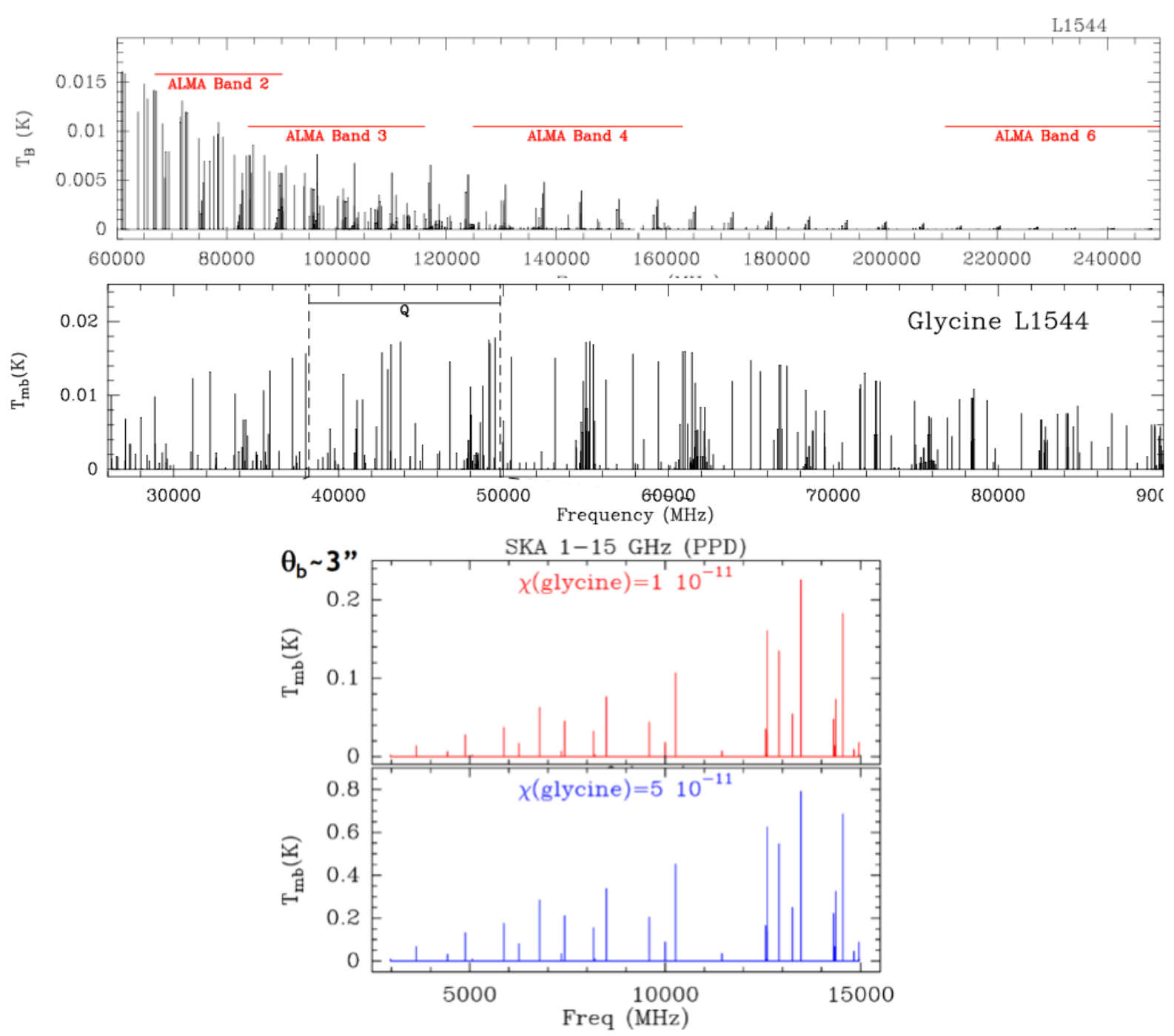

Figure 2: Top and middle panels: predictions for the Glycine rotational spectrum in a cold prestellar core (L1544); the spectrum shows the frequency ranges covered by the low frequency ALMA receivers (top) and the high frequency JVLA bands; the predicted intensities are low and difficult to reach even with these very sensitive facilities (adapted from the models of Jiménez-Serra et al. 2014). Bottom panel: preliminary preditions of Glycine line intensities in the frequency range from 1 through $15 \mathrm{GHz}$ covered by SKA1 for the TW Hya protoplanetary disk with two assumptions on the Glycine gas phase abundance (Jimenez-Serra et al., in preparation).

resolution, corresponding to typical disk sizes in the nearby star forming regions. This requirement is captured in the SKA1 science requirements (Braun 2014) which has been derived for prestellar cores, but can also apply to protoplanetary disks, scaling for the smaller expected size and narrower line. In fact, if the gas phase abundance of Glycine is on the high side of the expectated range, then the detection should not be very demanding and achievable with about $10 \mathrm{~h}$ on source. In addition, we remark that the simplistic assumption of local thermodynamic equilibrium for the line excitation, made in the computations, is very simplistic and may not apply to the conditions of these heavy molecules. The detection and analysis of Carbodiimide (HNCNH) in Sgr B2 has 
shown that several low frequency lines may be masing, increasing their chances for detection, but significantly complicating the physical modeling and interpretation (McGuire et al. 2012). It is important to note that these computations were made with the (ambitious) goal of detecting Glycine, it is expected that more abundant and less complex organic molecules will be easier to detect; note however that this field is still in its infancy as no complex organic molecule has yet been detected in protoplanetary disks. While ALMA is already tranforming the chemical study of warm and young protostars (eg. Jørgensen et al. 2012; Sakai et al. 2014), it is the lower frequency regime, the one probed by SKA1 with Band 5 or by the as yet unfunded ALMA Band 1 and 2, that will allow us to investigate throughly the chemical complexity of cold protoplanetary disk midplanes. We note that while an SKA1 equipped with Band 5 will allow to detect complex organic (and possibly pre-biotic) molecules in disks in nearby star forming regions, the interpretations of these lines will not be straightforward (see eg. Faure et al. 2014) and significant effort should be spent on laboratory measurements as well as chemical and astrophysical modeling to make the most effective use of the observations. However, SKA1 may not be able to produce resolved images of these faint molecular species, but this will be possible with the full SKA.

\subsection{Imaging of complex organic molecules in disks beyond SKA1}

To study complex molecule chemistry and the variation of abundance as a function of distance from the central star and across the snowlines, it will be necessary to perform spatially resolved studies. These studies will require a significant improvement of the sensitivity coupled with a good imaging performance. One order of magnitude improvement in sensitivity will be required to be able to image the disks at $1^{\prime \prime}$ resolution, which will allow to probe the effect of the CO snowline in the nearest disks around relatively massive stars (2-4 $\mathrm{M}_{\odot}$ ). Two orders of magnitude increase will allow to perform a detailed analysis of the chemistry of the disk around solar mass young stellar objects, possibly resolving the inner planet formation regions at $0.3^{\prime \prime}$ resolution.

\section{The temperament of young stars and its effect on disks}

Young stellar objects with disks produce high energy radiation in the ultraviolet and X-rays, caused by the accretion of material from the disk to the young star and by the active chromospheres. $\mathrm{X}$-ray variability is thought to originate from flaring powered by reconnection events in the stellar magnetosphere and is known to be very variable in young stellar objects with disks (see e.g. the recent surveys in ONC, Taurus and Ophiuchus star forming regions: Grosso et al. 2005; Güdel et al. 2007; Pillitteri et al. 2010). The high energy radiation from the central star (either from accretion or chromospheric activity) is known to affect disk evolution (Gorti et al. 2009), and is also expected to affect disk gas and solids chemistry (eg. Ábrahám et al. 2009; Banzatti et al. 2012). A systematic study of the effect of this high-energy irradiation variability on the disks is lacking because of the difficulties to set up coordinated X-ray and millimetre/infrared campaigns.

Radio continuum observations in the $1-12 \mathrm{GHz}$ range is an important tool to study the long term effects of stellar radiation on the disk by constraining the photoevaporative winds (GalvánMadrid et al. 2014; Pascucci et al. 2011, 2012), and has also the potential of allowing us to execute coordinated studies for the effects of energetic flares on the disk chemistry. X-ray activity and flaring is also known to be connected with radio flaring, although the sensitivity of existing radio 
facilities is limited and only a fraction of the X-ray detected objects are also identified as radio flaring (eg. Forbrich et al. 2011; Forbrich \& Wolk 2013).

\subsection{The temperament of young stars and its effect on disks with SKA1}

To detect and characterize photoevaporative winds in nearby star forming regions, it is necessary to reach $\mu$ Jy sensitivities at 1-10 GHz (Pascucci et al. 2012; Galván-Madrid et al. 2014) at least over the areas of the nearby star forming regions in the Gould Belt (these requirements are well captured in the SKA Level 0 Science Requirements, Braun (2014)). In addition, to perform systematic studies of radio flares and coordinate these studies with complementary observations of the molecular and solid state features with ALMA and/or the ELTs, it will be necessary to reach high sensitivity at low to intermediate frequencies $(0.1-1 \mathrm{GHz})$ and have the ability to immediately trigger pointed followups on the flaring sources. These observations will be possible with SKA1.

\subsection{The temperament of young stars and its effect on disks beyond SKA1}

One possible important avenue to pursue these studies in the future will be through the resolved spatial and kinematical imaging of the HI radio recombination lines expected in the disk photoevaporative flows (Pascucci et al. 2012). To allow resolved imaging, high sensitivity and imaging performance at $0.1^{\prime \prime}$ angular resolution and $0.1 \mathrm{~km} / \mathrm{s}$ spectral resolution are required. For this purpose the full SKA will be needed. The radio recombination line studies could be carried out with a slightly relaxed sensitivity $(\sim 30-100 \mathrm{mK})$, but with an angular resolution improved by approximately a factor of 100 over SKA1. This will require at least two orders of magnitude improvement in the sensitivity. While millimetre arrays, and especially ALMA, will allow to access the higher frequency, and brighter radio recombination lines, the combination of sensitivity and angular resolution of these facilities may not be adequate for this study.

\section{Summary}

In this chapter we have illustrated which would potentially be the unique contributions of SKA1 for the study of protoplanetary disks and planet formation. An SKA1 equipped with the high frequency Band 5 and satysfying the current version of the science requirements would provide transformational results in this area, addressing the fundamental questions of the formation of the rocky cores of planets, of the degree of chemical complexity of ices in protoplanetary disks and of the effects of the young stars on the disk physical and chemical evolution. We also outline the limitations of SKA1 and the potential areas to be addressed by a significantly upgraded version of the SKA.

Acknowledgements. This work was partly supported by the Italian Ministero dell' Istruzione, Università e Ricerca through the grant Progetti Premiali 2012 - iALMA (CUP C52I13000140001). Part of this research was carried out at the Jet Propulsion Laboratory, California Institute of Technology, under a contract with the National Aeronautics and Space Administration.

\section{References}

Ábrahám, P., Juhász, A., Dullemond, C. P., et al. 2009, Nature, 459, 224 
Banzatti, A., Meyer, M. R., Bruderer, S., et al. 2012, ApJ, 745, 90

Banzatti, A., Testi, L., Isella, A., et al. 2011, A\&A, 525, A12

Beckwith, S. V. W., Henning, T., \& Nakagawa, Y. 2000, Protostars and Planets IV, 533

Beckwith, S. V. W. \& Sargent, A. I. 1991, ApJ, 381, 250

Beckwith, S. V. W., Sargent, A. I., Chini, R. S., \& Guesten, R. 1990, AJ, 99, 924

Birnstiel, T., Klahr, H., \& Ercolano, B. 2012, A\&A, 539, A148

Blum, J. \& Wurm, G. 2008, ARA\&A, 46, 21

Braun, R. 2014, "SKA1 Level 0 Science Requirements", Document number SKA-TEL.SCI-SKOSRQ-001, SKA Organisation

Caselli, P., Keto, E., Bergin, E. A., et al. 2012, ApJL, 759, L37

Cossins, P., Lodato, G., \& Testi, L. 2010, MNRAS, 407, 181

Dipierro, G., Lodato, G., Testi, L., \& de Gregorio Monsalvo, I. 2014, MNRAS, 444, 1919

Elsila, J. E., Glavin, D. P., \& Dworkin, J. P. 2009, Meteoritics and Planetary Science, 44, 1323

Faure, A., Remijan, A. J., Szalewicz, K., \& Wiesenfeld, L. 2014, ApJ, 783, 72

Forbrich, J., Osten, R. A., \& Wolk, S. J. 2011, ApJ, 736, 25

Forbrich, J. \& Wolk, S. J. 2013, A\&A, 551, A56

Galván-Madrid, R., Liu, H. B., Felice Manara, C., et al. 2014, A\&A, 570, L9

Glavin, D. P., Dworkin, J. P., Aubrey, A., et al. 2006, Meteoritics and Planetary Science, 41, 889

Gorti, U., Dullemond, C. P., \& Hollenbach, D. 2009, ApJ, 705, 1237

Grosso, N., Feigelson, E. D., Getman, K. V., et al. 2005, ApJS, 160, 530

Güdel, M., Briggs, K. R., Arzner, K., et al. 2007, A\&A, 468, 353

Guilloteau, S., Dutrey, A., Piétu, V., \& Boehler, Y. 2011, A\&A, 529, A105

Hogerheijde, M. R., Bergin, E. A., Brinch, C., et al. 2011, Science, 334, 338

Holtom, P. D., Bennett, C. J., Osamura, Y., Mason, N. J., \& Kaiser, R. I. 2005, ApJ, 626, 940

Jiménez-Serra, I., Testi, L., Caselli, P., \& Viti, S. 2014, ApJL, 787, L33

Jørgensen, J. K., Favre, C., Bisschop, S. E., et al. 2012, ApJL, 757, L4

McGuire, B. A., Loomis, R. A., Charness, C. M., et al. 2012, ApJL, 758, L33

Miotello, A., Testi, L., Lodato, G., et al. 2014, A\&A, 567, A32

Modica, P. \& Palumbo, M. E. 2010, A\&A, 519, A22

Muñoz Caro, G. M., Meierhenrich, U. J., Schutte, W. A., et al. 2002, Nature, 416, 403

O'dell, C. R., Wen, Z., \& Hu, X. 1993, ApJ, 410, 696

Pascucci, I., Gorti, U., \& Hollenbach, D. 2012, ApJL, 751, L42

Pascucci, I., Sterzik, M., Alexander, R. D., et al. 2011, ApJ, 736, 13

Pérez, L. M., Carpenter, J. M., Chandler, C. J., et al. 2012, ApJL, 760, L17

Pillitteri, I., Sciortino, S., Flaccomio, E., et al. 2010, A\&A, 519, A34

Pinilla, P., Birnstiel, T., Ricci, L., et al. 2012, A\&A, 538, A114

Pizzarello, S., Krishnamurthy, R. V., Epstein, S., \& Cronin, J. R. 1991, GeCoA, 55, 905

Podio, L., Kamp, I., Codella, C., et al. 2013, ApJL, 766, L5

Qi, C., Öberg, K. I., \& Wilner, D. J. 2013, ApJ, 765, 34

Ricci, L., Testi, L., Natta, A., Scholz, A., \& de Gregorio-Monsalvo, I. 2012, ApJL, 761, L20

Ricci, L., Testi, L., Natta, A., et al. 2014, ApJ, 791, 20

Sakai, N., Sakai, T., Hirota, T., et al. 2014, Nature, 507, 78

Shu, F. H., Adams, F. C., \& Lizano, S. 1987, ARA\&A, 25, 23 
Testi, L., Birnstiel, T., Ricci, L., et al. 2014, Protostars and Planets VI, in press, arxiv:1402.1354 Testi, L., Natta, A., Shepherd, D. S., \& Wilner, D. J. 2001, ApJ, 554, 1087

Testi, L., Natta, A., Shepherd, D. S., \& Wilner, D. J. 2003, A\&A, 403, 323

Williams, J. P. \& Cieza, L. A. 2011, ARA\&A, 49, 67

Wilner, D. J. 2004, NewAR, 48, 1363

Wilner, D. J., D’Alessio, P., Calvet, N., Claussen, M. J., \& Hartmann, L. 2005, ApJL, 626, L109 